\title{
Pyelonephritis xanthogranulomatosa in a 7-year-old girl
}

\author{
Sylwester Gerus ${ }^{1}$, Wojciech Apoznański ${ }^{1}$, Tomasz Szydełko², Dariusz Patkowski ${ }^{1}$
}

'Department of Pediatric Surgery and Urology, Wrocław Medical University, Wrocław, Poland

2Department of Urology and Oncological Urology, Wrocław Medical University, Wrocław, Poland

\section{KEY WORDS}

xanthogranulomatous pyelonephritis

nephrectomy histopathological evaluation

\section{ABSTRACT}

\begin{abstract}
Xanthogranulomatous pyelonephritis (XGPN) is rare form of chronic, usually unilateral, renal infection that involves damage to the renal glomeruli and periglomerular tissue and destruction of the renal parenchyma. Nephrectomy is usually necessary and the prognosis is good if XGPN is unilateral and treated early. XGPN typically affects middle-aged females and is extremely rare in children. We report one case of XGPN in a seven-yearold girl who was operated on at our institution be-cause of lower urinary tract dysfunction and renal insufficiency - the left kidney was removed and the bladder was augmented by means of the remaining ureter. The histopathological evaluation of the removed kidney showed chronic XGPN
\end{abstract}

\section{INTRODUCTION}

Xanthogranulomatous pyelonephritis (XGPN) is a rare form of chronic, usually unilateral, renal infection (about 300 cases have been reported since 1916), which involves damage to the renal glomeruli and periglomerular tissue as well as the destruction of the renal parenchyma, which is replaced by granulomatous tissues $[1,2]$.

Nephrectomy is usually necessary and the prognosis is good if XGPN is treated early enough and the other kidney is not affected [3]. XGPN typically affects middle-aged females [4] and is extremely rare in children (the first case was diagnosed in 1963[5]). We report one case of XGPN in a seven-year-old girl who was operated on at our institution because of lower urinary tract dysfunction and renal insufficiency. In the course of the operation the left kidney was removed and the bladder was augmented by means of the remaining ureter. The histopathological evaluation of the removed kidney showed chronic XGPN.

\section{CASE REPORT}

A seven-year-old girl was admitted to our institution from the Pediatric Nephrology Clinic with the diagnosis of neurogenic voiding dysfunction, acute pyelonephritis, right hydronephrosis, left vesicoureteral reflux and left renal hypofunction. The first urinary tract infection had been recognized when the girl was seven months old. Then urine culture had shown Entercoccus sp. Another infection was diagnosed four months later and the girl was treated at a pediatric ward. Blood test, urinalysis, urine culture, and ultrasound of the abdomen revealed no abnormalities. There were no further episodes of urinary tract infection.

The girl's mother referred to a general practitioner with her 6-year-old daughter complaining of nocturnal enuresis. Ultrasound revealed that the left kidney was significantly enlarged, as were the pelvicalyceal system and the proximal part of the ureter. Urine retention (about $50 \mathrm{ml}$ ) after micturition was also observed. Numerous diverticuli in the bladder wall were noticed. The girl was sent to the Clinic of Pediatric Nephrology, Wrocław for diagnosis and treatment. On admission, the girl's condition was regarded as stable and physical examination revealed no abnormalities. Blood test, electrolytes, creatinine, C-reactive protein, and total protein serum concentration were normal. Only the level of alkaline phosphatase was above the norm. Urinalysis indicated an increased number of leucocytes and urine culture was negative.

Imaging studies revealed complex dysfunction of the urinary tract including the diverticular bladder, left vesicoureteral reflux, bilateral hydronephrosis and significant functional insufficiency of the left kidney.

Urography: neurogenic voiding dysfunction; bilateral hydronephrosis, which was larger on the left side; left renal hypofunction; layering of the contrast medium in the kidney, visible after 6 hours and traceable after 9 hours.

Cystography: an irregular outline of the bladder; numerous diverticuli of the bladder; left vesicoureteral reflux suspected; a normal urethra.

Tc99m-EC (Ethylenedicysteine) scintigraphy: left kidney - 19\%, right kidney - $81 \%$ of total renal function; numerous post-infective scars in the left kidney.

Cystometry: bladder capacity - $181 \mathrm{ml}$; intravesical pressure at the end of the filling phase $-131 \mathrm{~cm} \mathrm{H}_{2} \mathrm{O}$ without urine leakage; reduced bladder compliance. Oxybutynin and clear intermittent catheterization $(\mathrm{CIC})$ were recommended.

On January 25, 2008, the girl referred to the Clinic of Pediatric Nephrology again with symptoms of a urinary tract infection. On admission the patient was visibly suffering, pale, weak, sleepy, and dehydrated. Pain of the abdomen and bilaterally positive Goldflam sign were noted on the physical. Urine culture yielded Pseudomonas aeruginosa. The laboratory showed unbalanced metabolic acidosis, elevation of serum creatinine concentration (3.41 mg\%, $3.52 \mathrm{mg} \%$ ) and of C-reactive protein ( $72.9 \mathrm{mg} / \mathrm{l} ; 23.1 \mathrm{mg} / \mathrm{l}$ ), and anemia (hemoglobin 8.4 g\%, 6.6g\%, $11.4 \mathrm{~g} \%)$. Urinalysis showed albuminuria and massive pyuria. Ultrasound of the abdomen indicated progressive enlargement of the pelvicalyceal systems of both kidneys as well as enlargement of the left ureter along its entire length. The following procedures were applied: parenteral hydration, intravenous antibiotic therapy, erythrocyte transfusion, and clean intermittent catheterization (CIC). As a result, the child's condition improved together with the biochemical parameters. Control urine culture was sterile. However, after 10 days the girl's condition deteriorated 
again. Fever and massive pyuria were noted. Urine culture yielded Proteus mirabilis. Urography showed no contrast secretion on the left. The patient was subjected to an operation in the Children's Clinic of Surgery and Urology, Wrocław Medical University. Right laparoscopic nephrectomy was carried out, the ureter was released, and augmentation of the bladder was performed using the ureter in order to increase the bladder capacity (ureterocystoplasty). The patient had an uneventful postoperative recovery and the girl was discharged from hospital in a good physical condition.

She was instructed to regularly empty the bladder by $\mathrm{ClC}$ and to regularly refer to a nephrologist. The histopathological evaluation of the removed kidney showed XGPN.

Currently the girl is under nephrological and urological surveillance in our institution. Her latest visit indicated proper psycho-physical development and no urinary tract infection since the surgery.

\section{DISCUSSION}

XGPN is a rare form of chronic, usually unilateral, renal infection. Since 1916, when Schlagnehaufter described the first case [6], 300 cases have been reported. The etiology of the disease remains unknown. It typically affects middle-aged women [4]. In children it is extremely rare [5]. Our case involves a seven-year-old girl with recurrent urinary tract infections. Urinary tract infection, particularly if induced by Proteus sp., is believed to predispose patients to the disease. What might have influenced the clinical course of the disease in our case was neurogenic dysfunction of the lower urinary tract, which was revealed on radiology and cystometry.

However, the cause of the above change was not determined. Neither nuclear magnetic resonance (NMR) of the spinal cord nor neurological examination revealed any damage to the central nervous system (CNS), which in theory might have been the cause of the neurogenic voiding dysfunction of the lower urinary tract.

We should mention the poor social conditions the child comes from. She was born prematurely ( $36^{\text {th }}$ week of pregnancy) to a large family in a small poverty-stricken village.

A thorough analysis of our case confirms that the disease has no clear clinical image. XGPN has been described as "the great imitator" because, in its symptoms, it is similar to renal tumors, numerous renal abscesses with abscesses of other organs, and to urosepsis. Asymptomatic bacteriuria may be noted and the symptoms may be suggestive of urinary tract infection $[5,7]$ What seems characteristic of XGPN is the progression of changes with recurrent urinary tract infections becoming more frequent. As our patient had turned 2-years-old, she had urinary tract infections recognized every now and then, and immediately before the operation they became serious, with symptoms typical of urosepsis. An additional complication in this case was neurogenic dysfunction of the urinary tract with left vesicoureteral reflux. It has not been determined yet if there was any correlation between the above afflictions and XGPN. What we know is that they contributed, together with the infection, to the deterioration of renal function, which eventually became nonfunctional. As a consequence, nephrectomy and ureterocystoplasty had to be performed - nephrectomy because of recurrent, persistent urinary tract infections with sepsis and accompanying left vesicoureteral reflux, and ureterocystoplasty in order to increase bladder capacity thereby decreasing bladder pressure. Previous conservative treatments had been unsuccessful. Antibiotic therapies had eliminated the infection for some time, but it recurred soon after with a more sever course. The above observations corroborate with that which was previously reported. The unanimously shared view is that only surgery is effective and can lead to recovery. Since the operation no urinary tract infections have been recognized in our patient. She grows and develops properly. Interestingly, her cystometric record has been back to normal. She urinates properly. No urine retention after micturition is observed so catheterization is unnecessary. The question arises then whether the lower urinary tract dysfunction was mimicked by XGPN.

\section{CONCLUSIONS}

We believe that our case report has shown that, rare as it is, XGPN has to be borne in mind not only when middle-aged women are being diagnosed but also whenever a patient has nonspecific symptoms of a urinary tract disease. It is noteworthy, however, that only histopathological evaluation is capable of providing definitive diagnosis, although some authors suggest preoperative fine-needle aspiration cytology might help to diagnose the condition earlier (in our case fine-needle aspiration biopsy was not performed because the left kidney was nonfunctional and had to be removed regardless of the reason).

\section{REFERENCES}

1. Sugie $S$, Tanaka $T$, Nishikawa $A$, et al: Fine-needle aspiration cytology of xanthogranulomatous pyelonephritis. Urology 1991; 37: 376-379.

2. Bingol-Kologlu M, Ciftci AO, Senocak ME, et al: Xanthogranulomatous pyelonephritis in children: diagnostic and therapeutic aspects. Eur J Pediatr Surg 2002; 12: 42-48.

3. Alan C, Ataus S, Tunc B: Xanthogranulomatous pyelonephritis with psoas abscess: 2 cases and review of the literature. Int Urol Nephrol 2004; 36: 489-493.

4. Chuang CK, Lai MK, Chang PL, et al: Xanthogranulomatous pyelonephritis: experience in 36 cases. J Urol 1992; 147: 333-336.

5. Schlagenhaufter F: Uber eigen tumliche staphylomykosen der nieren and des para renalen Bindegewebes. Frankfurt Ztschr Path 1916; 19: 139-148.

6. Zorzos I, Moutzouris V, Petraki C, Katsou G: Xanthogranulomatous pyelonephritis--the "great imitator" justifies its name. Scand J Urol Nephrol 2002; 36: 74-76.

7. Hendrickson RJ, Lutfiyya WL, Karrer FM, et al: Xanthogranulomatous pyelonephritis. J Pediatr Surg 2006; 41: 15-17.

\section{Correspondence}

Sylwester Gerus

Department of Pediatric Surgery and Urology

50-369 Wrocław, Poland

ul. M. Skłodowskiej-Curie 52

phone: +48 606369311

sylwestergerus@gazeta.pl 